\title{
Modelo de calidad para el mejoramiento de la eficiencia en las instituciones públicas del Ecuador
}

\author{
Quality model for improving efficiency in Ecuador's public institutions
}

César Iván Casanova Villalba. ${ }^{1}$, Maybelline Jaqueline Herrera Sánchez. ${ }^{2}$ Cecilia Mercedes Navarrete Zambrano. ${ }^{3}$, \& Silvana Elizabeth Ruiz López. ${ }^{4}$

Recibido: 12-10-2020 / Revisado: 16-11-2020 /Aceptado: 05-12-2020/ Publicado: 02-01-2021

\begin{abstract}
.
DOI: $\underline{\text { https://doi.org/10.33262/cienciadigital.v5i1.1516 }}$

Introduction: Every public institution has a mission to provide quality care to the citizenry, but variations or limitations such as budget, accessibility, technological difficulties, among other factors hinder the efficiency of the institutions, which is why strategies must be sought to promote continuous improvement despite these difficulties. Objective: To present a quality model for improving the efficiency of Ecuador's public institutions, a model that takes into account the adversities these institutions are going through and that cannot incur large investments. Methodology: The model takes theoretical and historical research, so it will be a quantitative research when evaluating the situations faced by the public sector, but it will also be quantitative when trying to standardize with statistical models the results. The research will begin with the survey of the structure of the root problems through the application of surveys

${ }^{1}$ Universidad Técnica Luis Vargas Torres, Facultad de Ciencias Administrativas y Económicas, Sede Santo Domingo, Ecuador, cesar.casanova.villalba@utelvt.edu.ec, ID de ORCID https://orcid.org/0000-0001-

${ }^{2}$ Universidad Técnica Luis Vargas Torres, Facultad de Ciencias Administrativas y Económicas, Sede Santo Domingo, Ecuador, maybelline.herrera.sanchez@utelvt.edu.ec, ID de ORCID 0000-0001-6840-3891

${ }^{3}$ Universidad Técnica Luis Vargas Torres, Facultad de Ciencias Administrativas y Económicas, Sede Santo Domingo, Ecuador, cecilia.navarrete@utelvt.edu.ec, ID de ORCID 0000-0002-2953-3279

${ }^{4}$ Universidad Técnica Luis Vargas Torres, Facultad de Ciencias Administrativas y Económicas, Sede Santo Domingo, Ecuador, silvana.ruiz.lopez@utelvt.edu.ec, ID de ORCID 0000-0002-8211-3556
\end{abstract} 6486-1334 
and interviews, forming a fishbone, and be analyzed with the Pareto diagram. Results: To present a model of continuous improvement that drives to improve the institutional image, but without incurring in too high investments that affect the budgets deducted with which the entities of the public sector count, the model will be focused in instruments of quality of zero cost like the $5 \mathrm{~s}$, identification and elimination of mudas, training and control of production either of products and services that the entity delivers. Conclusions: The public sector entities have always been seen as inferior based on different scenarios, but always catalogued as inefficient. The model presented seeks to change this paradigm so that the improvement does not entail expenses that are outside the pre-established budgets.

Keywords: Quality Model, Continuous Improvement, Efficiency, Public Sector.

\section{Resumen.}

Introducción: Toda institución pública tiene en su misión el brindar atención de calidad a la ciudanía, pero variantes o limitantes como presupuesto, accesibilidad, dificultades tecnológicas, entre otros factores entorpecen la eficiencia de las instituciones, motivos por el cual se deben buscar estrategias que impulsen la mejora continua pese a estas dificultades. Objetivo: Presentar un modelo de calidad para el mejoramiento de la eficiencia de las instituciones públicas del Ecuador, modelo que considera las adversidades por las que atraviesan dichas instituciones y que no pueden incurrir en grandes inversiones. Metodología: El modelo toma investigaciones teóricas e históricas, por lo cual será una investigación cuantitativa al momento de evaluar las situaciones que enfrenta el sector público, pero también será cuantitativa al momento de tratar de estandarizar con modelos estadísticos los resultados. La investigación tendrá su inicio con el levantamiento de la estructura de los problemas raíz mediante la aplicación de encuestas y entrevistas, plasmando en una espina de pescado, y ser análisis con el diagrama de Pareto. Resultados: Presentar un modelo de mejora continua que impulse a mejorar la imagen institucional, pero sin incurrir en inversiones demasiado elevadas que afecten a los presupuestos deducidos con los que cuentan las entidades del sector público, se focalizaran el modelo en instrumentos de calidad de costo cero como las $5 \mathrm{~s}$, identificación y eliminación de mudas, capacitación y control de producción ya sea de productos y servicios que entregue la entidad. Conclusiones: Las entidades del sector público siempre han sido vistas de manera inferior esto en base a diferentes escenarios, pero siempre catalogado como ineficiente, mediante el modelo presentado, lo que se busca se cambie ese paradigma y que la mejora no conlleve gastos que estén fuera de los presupuestos preestablecidos.

Palabras claves: Modelo de Calidad, Mejora continua, Eficiencia, Sector público. 


\section{Introducción.}

Las personas siempre buscamos un producto o servicio que satisfaga nuestra necesidad, pero en la actualidad ya no basta solo con tener el producto, sino que ahora se valora la calidad tanto del bien o servicio como la calidad en todo el proceso de adquisición. Según Rueda (2011) la ciudadanía se interesa por saber en qué se destinan los impuestos que pagan, por tal motivo relacionan a mayor cantidad mejor imagen institucional, pero a esto se agrega una variante, que es percibida como la calidad que debe ser constante, y la misma debe manifestarse en todos los niveles del sector público; sean estas instituciones de nivel central, ejecutivo desconcentrado, empresas públicas o gobiernos autónomos.

Con este antecedente la responsabilidad de todo gobierno es mejorar los servicios o productos que oferta mediante sus instituciones públicas, por lo tanto, es imprescindible la mejora continua, en palabas de Casanova (2018) la mejora continua es la aplicación de varias herramientas con un mismo fin, el cual es planificar, hacer, controlar y mejorar continuamente los elementos o actividades para cumplir con las expectativas de los ciudadanos.

Pese a que exista la necesidad por los ciudadanos y la obligación por parte del estado, en mejorar la calidad de los servicios públicos, se debe considerar que una mejora por lo regular siempre contrae un mayor gasto público, es decir las instituciones del sector público incrementan sus gastos, pero a la par se debe incrementar la calidad de los productos y servicios ofertados. (Rueda López, 2011)

Utilizando la ideología de Herrera (2018) la cual establece que para mejorar la satisfacción de los ciudadanos es necesario incrementar la productividad, y que la misma se debe analizar desde la recepción hasta la entrega del producto final, la presente investigación busca presentar un modelo de mejora continua, el cual pueda ser plasmado dentro de las instituciones públicas, pero sin afectar a sus presupuestos.

\section{Base legal en Ecuador para mejorar la calidad en las instituciones publicas}

La importancia de un modelo para mejorar la eficiencia del sector público se encuentra fundamentado en la Constitución del Ecuador, ya que en la misma se indica "las personas tienen derecho a recibir bienes y servicios de óptima calidad" (2008), en este enunciado se deja claro que las entidades públicas deben ser entregar productos o servicios de calidad, y de no cumplir con esta disposición, la misma Constitución del Ecuador (2008) establece el "control de calidad y los procedimientos de defensa de las consumidoras y consumidores; y las sanciones por vulneración de estos derechos, la reparación e indemnización por deficiencias, daños o mala calidad de bienes y servicios". 
Con este antecedente en el país se genera varias normativas adicionales que tratan el tema de la mejora continua y la calidad de los servicios, mismos que se detallan en la tabla 1 a continuación:

Tabla 1: Resumen base legal de la calidad en el sector publico

\begin{tabular}{|c|c|c|c|c|}
\hline Normativa & Registro 0 & ficial & Fecha & Observación \\
\hline $\begin{array}{l}\text { Ley Orgánica para la Optimización y } \\
\text { Eficiencia de Trámites Administrativos }\end{array}$ & $\begin{array}{l}\text { Suplemento } \\
353\end{array}$ & Nro. & $\begin{array}{l}23 \text { de octubre } \\
\text { de } 2018\end{array}$ & Art. 22 \\
\hline $\begin{array}{l}\text { Reglamento General a la Ley Orgánica } \\
\text { para la Optimización y Eficiencia de } \\
\text { Trámites Administrativos }\end{array}$ & $\begin{array}{l}\text { Suplemento } \\
505\end{array}$ & Nro. & $\begin{array}{l}10 \text { de junio de } \\
2019\end{array}$ & Art. 11 \\
\hline Plan Nacional de Desarrollo 2017 - 2021 & N/A & & N/A & Objetivo 7 \\
\hline $\begin{array}{l}\text { Norma Técnica del Subsistema de } \\
\text { Evaluación del Desempeño }\end{array}$ & $\begin{array}{l}\text { Suplemento } \\
218\end{array}$ & Nro. & $\begin{array}{l}10 \text { de abril del } \\
2018\end{array}$ & Art 15 \\
\hline $\begin{array}{l}\text { Norma Técnica de los Mecanismos de } \\
\text { Calificación del Servicio }\end{array}$ & $\begin{array}{l}\text { Suplemento } \\
45\end{array}$ & Nro. & $\begin{array}{ll}23 & \text { de } \\
\text { septiembre } & \text { de } \\
2019 & \end{array}$ & N/A \\
\hline
\end{tabular}

Fuente: Subsecretaría de Calidad en el Servicio Público (2020)

\section{Análisis situacional}

La Constitución del Ecuador (2008), en su artículo 227 dispone "la administración pública constituye un servicio a la colectividad que se rige entre otros por los principios de eficacia, eficiencia, calidad", la misma constitución plasma en el artículo 52 "las personas tienen derecho a disponer de bienes y servicios de óptima calidad". Para lo cual estableceros en primera instancia a que se denomina sector público, según el Instituto Nacional de Estadísticas y Censos (INEC):

Es el conjunto de prestaciones de interés público que son otorgadas por parte del Estado hacia la sociedad. De acuerdo a la Constitución, el Estado será responsable de la provisión de los servicios públicos de agua potable y de riego, saneamiento, energía eléctrica, telecomunicaciones, vialidad, infraestructuras portuarias y aeroportuarias, y los demás que determine la ley. (2018)

Con este enfoque se detalla que todos los serivicios básicos, seguridad, y accesibilidad conforman el sector público de un país, por ende todas estas entidades deben prestar servicios de calidad. Según datos del INEC obtenidos de la encuesta multiproposito a los hogares bajo el objetivo 7 (Objetivo delimitados por el Plan Nacional de Desarrollo), en su indicador Índice de percepción de atención y calidad del servidor público, nos da una imagen general 
de la percepción de la ciudadanía frente a la calidad de los servicios públicos, datos que presentamos de manera resumida en la Figura 1:

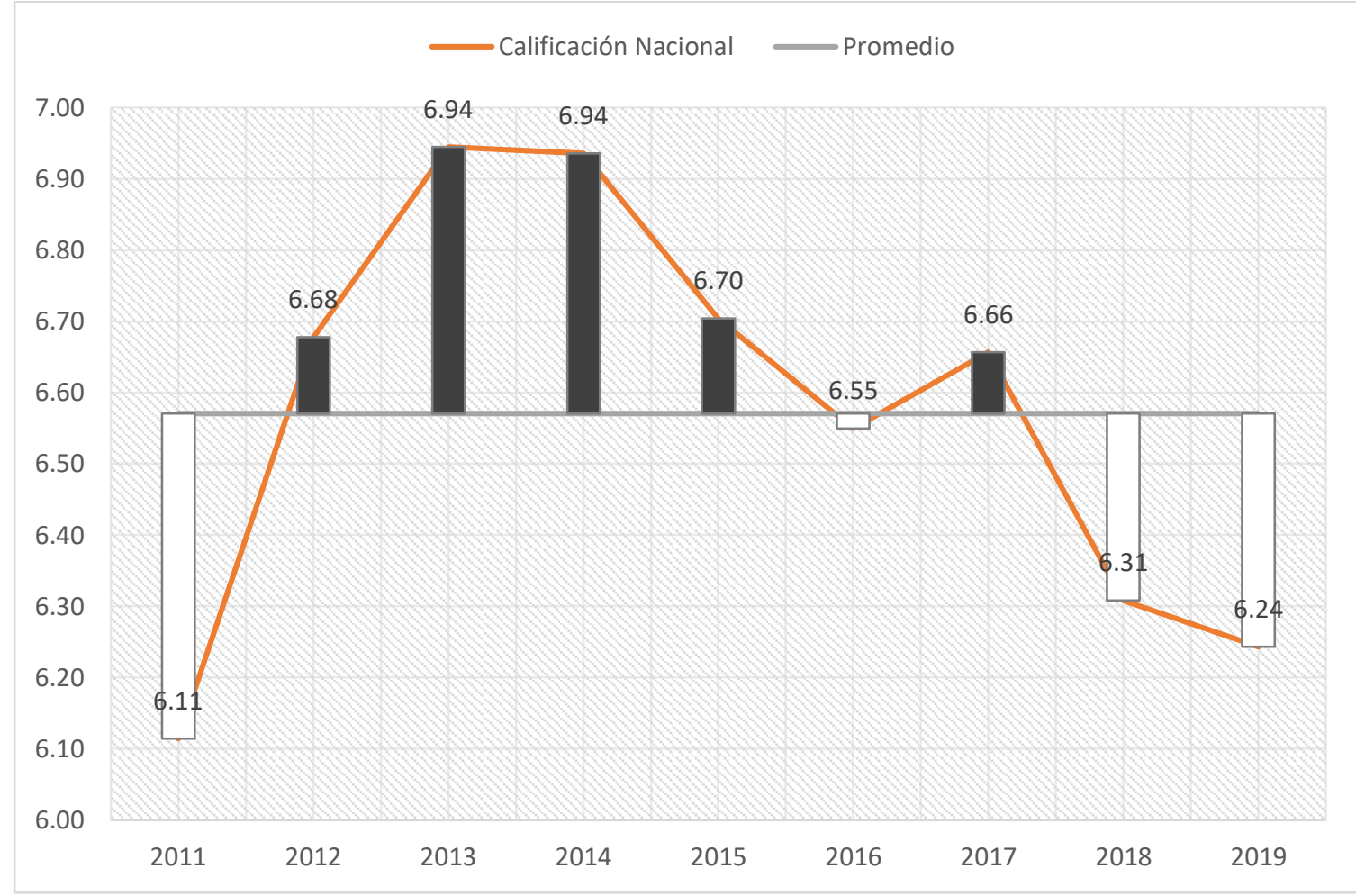

Figura 1: Percepción de la calidad de los servicios públicos en general (2013-2019)

Fuente: Instituto Nacional de Estadística y Censo (2020)

Este censo por parte del INEC es realizado a nivel nacional y su ponderación es sobre 10, lo que refleja que para el 2019 el Ecuador tiene una percepción de calidad de los servicios públicos de 6,24, una calificación baja en comparación a la de la región. Motivos por los cuales se deben implementar estrategias en cada institución y lograr incrementar este indicador por medio de la mejora continua.

\section{Factores críticos}

Análisis PEST. - Un análisis político, económico, social y tecnológico (PEST), según Casanova (2018) refleja las variables externas que afectan a las instituciones públicas o privadas, a continuación, en la tabla 2 , se presenta un análisis general: 
Tabla 2: Análisis PEST

\begin{tabular}{|c|c|}
\hline Factor & Análisis \\
\hline Político & $\begin{array}{l}\text { Las instituciones públicas independientemente de su nivel o naturaleza, } \\
\text { están atadas a los cambios políticos de cada gobierno de turno, a esto } \\
\text { se suman las situaciones no éticas en general. }\end{array}$ \\
\hline Económico & $\begin{array}{l}\text { Al depender de asignaciones presupuestarias del estado, los recursos } \\
\text { van a estar delimitados estrictamente por el tema político y adicional a } \\
\text { esto por temas coyunturales. }\end{array}$ \\
\hline Social & $\begin{array}{l}\text { El ámbito social afecta a los intereses del estado, no responde a } \\
\text { necesidades específicas o de impacto nacional, por el contrario, es } \\
\text { afectado por la presión social en un determinado tiempo, por ende, el } \\
\text { accionar de las instituciones va a estar ligado a la presión social e } \\
\text { intereses del gobierno }\end{array}$ \\
\hline Tecnológico & $\begin{array}{l}\text { El factor tecnológico es una variable que pese a ser una de las más } \\
\text { importantes para la mejora continua, no es tomada en consideración } \\
\text { debido a la afectación que conlleva a la inversión institucional, es decir } \\
\text { al gasto de recursos. }\end{array}$ \\
\hline
\end{tabular}

Fuente: Elaboración propia

\section{Metodología.}

Las empresas sean públicas o privadas e indistintamente de su tamaño deben implementar mejora continua, la ISO 9000 (2015) la define como la actividad recurrente para mejorar el desempeño, o desde el punto de vista de Imai (1991) es un proceso de mejora incremental, continuo, sistemático y ordenado.

La metodología aplicada en la presente investigación está enfocada en el pensamiento Kaizen que según Becerra (2013) significa mejoramiento continuo tanto en la vida profesional, familiar social y laboral, es decir involucra a todo por igual. Por lo tanto, la investigación está basada en estudios teóricos e históricos de datos recabados por autores de calidad, para ser plasmado en datos cuantitativos y cualitativos, dando una investigación mixta que trata de manera deductiva los principios de calidad y leyes emanadas para ser estudiadas de manera particular los hechos en el sector público.

Para la presentación del modelo de mejora se inicia con la metodología de Gobierno por Resultados (GPR), el cual permite gestionar de manera eficiente los planes estratégicos, operativos, proyectos entre otros y colabora en el control en los distintos niveles obteniendo resultados mediante indicadores, con la utilización de herramientas que permiten guiar las acciones del gobierno a los objetivos planteados. (Secretaría Nacional de la Administración Pública, 2011) 
Con el enfoque de GPR, se estructura el modelo adicionando la metodología Deming que es la utilización Planificar, Hacer, Verificar y Actuar PHVA, centrándose en la ideología de las 5s, para Masaaki Imai (2012), con sus nombres en japonés se detallan en la siguiente tabla 3:

Tabla 3: 5’S

\begin{tabular}{|c|c|c|}
\hline Japones & Significado & Ideología \\
\hline Seiri & $\begin{array}{l}\text { Solo lo } \\
\text { necesario }\end{array}$ & $\begin{array}{l}\text { Según un análisis realizado por Casanova (2018), se encuentra } \\
\text { común que en las instituciones se encuentran documentos sin un } \\
\text { orden de importancia o urgencia, exista desorden y perdida de } \\
\text { documentos. } \\
\text { Seiri busca que tengamos solo lo necesario en nuestro espacio de } \\
\text { trabajo. }\end{array}$ \\
\hline Seiton & Ordenar & $\begin{array}{l}\text { Cuando tenemos demasiados documentos y no han sido } \\
\text { catalogados los que son necesarios para la tarea a ejecutar, no } \\
\text { existe un orden. } \\
\text { Seiton busca que exista un orden de los documentos que tenemos } \\
\text { en nuestro espacio, de lo más importante y necesario. }\end{array}$ \\
\hline Seiso & Limpiar & $\begin{array}{l}\text { Cuando tengamos lo necesario en nuestro puesto de trabajo y } \\
\text { ordenado, lo siguiente es mantener limpio nuestro espacio de } \\
\text { trabajo lo que se traduce en Seiso. }\end{array}$ \\
\hline Seiketsu & Seguridad & $\begin{array}{l}\text { Seiketsu es la seguridad de mantener los tres pasos anteriores en } \\
\text { cada momento y trabajar con la seguridad que la clasificación de } \\
\text { necesario, ordenado y limpieza me impulsa a ser más productivo } \\
\text { y disminuir los errores. }\end{array}$ \\
\hline Shitsuke & Disciplina & $\begin{array}{l}\text { Por último, Shirsuke es la auto disciplina, que significa que esto } \\
\text { ya sea una rutina o cultura de trabajo, pero no solo en lo laboral } \\
\text { si no en lo personal, familiar, en todo momento y en cada } \\
\text { actividad realizada. }\end{array}$ \\
\hline
\end{tabular}

Fuente: Masaaki Imai (2012)

Un resumen de las 5's según Casanova (2018), manifiesta que se debe "descartar lo que no necesitamos (seiri), ordenando todos los elementos (seiton). Entonces se debe mantener un ambiente limpio para que podamos identificar fácilmente las anomalías (seiso), y estos se deben mantener de forma continua (shitsuke), en conjunto la autodisciplina (Shitsuke)".

Una vez identificado la base del modelo es necesario conocer el ciclo PHVA, o plan-docheck-act (PDCA) en inglés, la metodología para mejorar continuamente y su aplicación resulta muy útil en la gestión de los procesos. (Camisón, Cruz, \& González, 2006), se resume el ciclo indicando que se debe planificar las actividades a realizar previamente con lo que se disminuyen errores dentro del proceso ya que las personas saben que y como deben hacer su trabajo, pasamos a la segunda fase que es hacer, en esta como se tiene una guía que fue la 
planificación se ejecutan las actividades pero siempre puede existir inconvenientes o novedades por lo cual viene la etapa de control o verificación la cual se mide a través de indicadores, y en base a estos resultados se plantean las acciones, nos encontramos en la cuarta fase la cual es plantear soluciones a los inconvenientes y que para el nuevo ciclo se mejore.

\section{Costos de no conformes}

La mejora continua ademar se mejorar la imagen institucional con la satisfacción de los ciudadanos, reduce los costos de no calidad (Colunga, 1994), realizar un análisis de los defectos en términos económicos es de vital importancia para delimitar metas de reducción de desperfectos y por ende los costos de la no calidad. (Camisón, Cruz, \& González, 2006).

De manera general los costos de no conformes en el sector público tienen demasiadas connotaciones dependiendo el accionar de la institución, pero tomando la metodología de Casanova (2018) diremos de manera general que se pueden categorizar en dos, los internos que podrán ser suministros, mano de obra para corregir los errores, sanciones impuestas, entre otras la segunda parte estará representada por los efectos de ese producto o servicio no conforme como accidentes, daños materiales y en el peor de los casos pérdidas humanas.

Dependiendo de la institución se deberá implementar indicadores o metodologías de cálculo para obtener costos de no conformes, puedan ser estos por detección de Mudas, errores, reprocesos, compensaciones por errores cometidos, compensaciones por productos defectuosos entre otros; esto es en base a la misión de la entidad, pongamos como ejemplo a una institución que se encargue de un servicio básico como la electricidad, la suspensión del servicio aunque sea por minutos representa perdidas de recaudación, perdidas de producción a las empresas, posiblemente pérdidas humanas si trasciende a hospitales, estos enfoques se deben considerar para los costos de no conformes o de falta de calidad.

Delimitar los costos de la baja calidad nos evita incurrir en mayores gastos por reingeniería, en palabras de Casanova (2014)“el replanteamiento fundamental y rediseño radical de los procesos de las empresas para conseguir mejoras sustanciales en medidas de desempeño contemporáneas tan decisivas como costos calidad, servicio y rapidez". Según Herrera (2014) el estado invierte en lo que se refiere a responsabilidad social, por ende, genera entes de control y reguladores que controlen a las demás instituciones gubernamentales que brinden un servicio de calidad a la sociedad.

\section{Resultados.}

Como objetivo de la presente investigación se estableció el presentar un modelo de mejora que este encabezado por la estructura GPR y por ende pueda ser controlado por el ente de control respectivo, a lo cual se adiciono el ciclo de mejora continua PHVA centrado en la 
ideología de las 5’s, pero que el modelo no represente afectación al presupuesto de las instituciones, en tal virtud se presenta la Figura 2 como modelo de mejora:

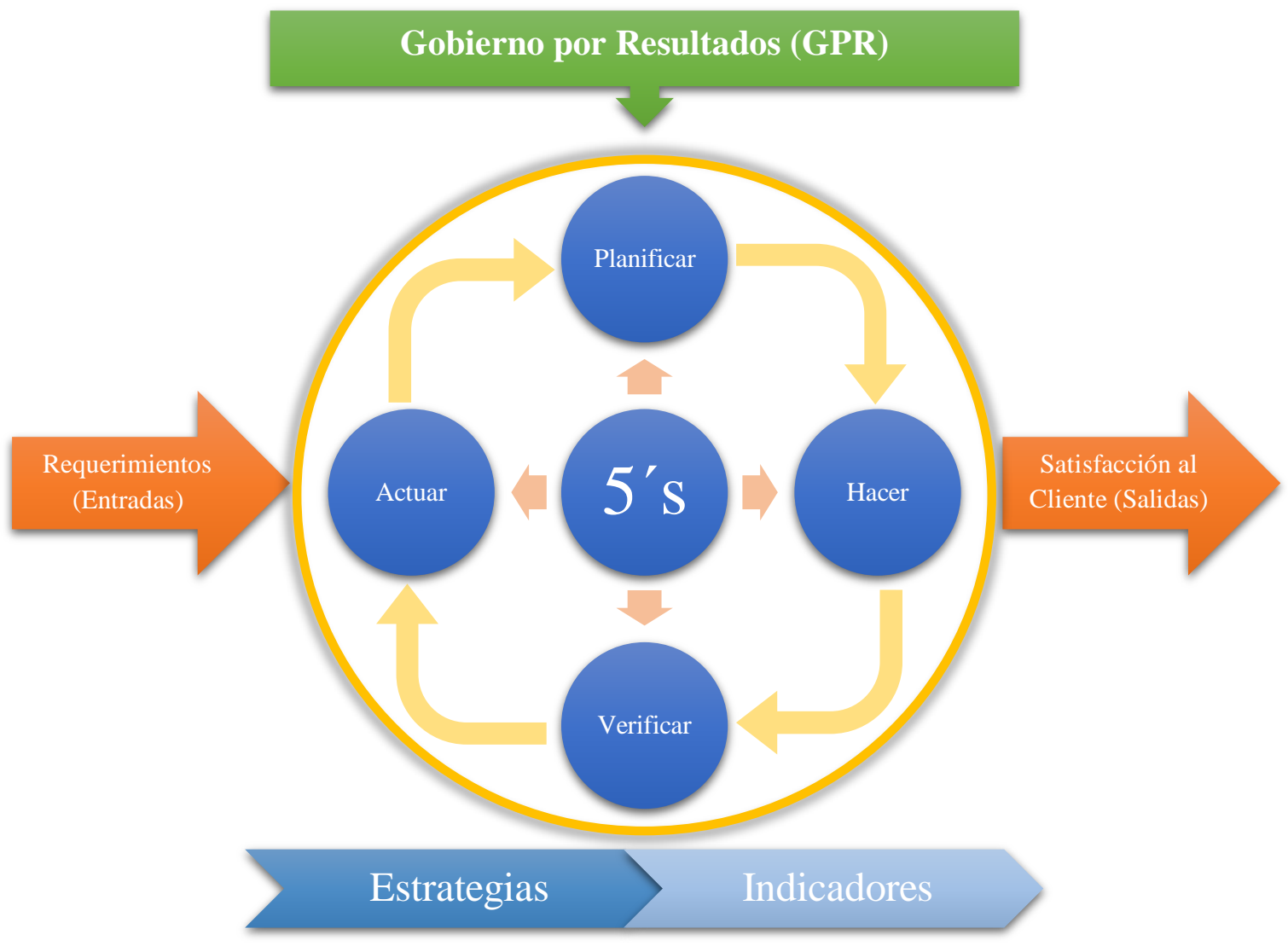

Figura 2: Modelo de mejora continua sector público

Fuente: Casanova (2018)

\section{Requerimientos (Entradas)}

Los requerimientos son los productos o servicios que oferta la institución, para que el modelo pueda tener efecto se recomiendo en primera instancia delimitar claramente los tipos de productos y servicios, ya que el modelo funciona en un proceso productivo, si se mesclan los procesos no tendrá el mismo efecto ya que las tareas intermedias pueden no ser claras o pueden confundirse en el tipo de proceso. Dentro de las instituciones del sector público se tiene la facilidad que todo está claramente normado, por lo cual se debe recurrir a las normas, reglamentos, leyes, y demás normativa con la finalidad de delimitar cada producto con su debido proceso.

\section{PHVA}

Deming presentó el ciclo PDCA en los años 50 en Japón, aunque señaló que el creador de este concepto fue W. A. Shewhart, quien lo hizo público en 1939, por lo que también se lo denomina «ciclo de Shewhart»o «ciclo de Deming» indistintamente (Ishikawa, 1986). Este 
ciclo es muy antiguo, pero en la actualidad sigue siendo utilizado y con resultados de alto impacto en las instituciones.

Planificación: dentro de esta actividad para la aplicación del modelo se mencionan las requisitos o actividades a ser efectuadas para la implementación y levantamiento de la situación inicial de la institución, recordando que se debe hacer a un proceso en específico. Las actividades se detallan en la tabla 4:

Tabla 4: Actividades de la planificación según el modelo de mejora

\begin{tabular}{|c|c|}
\hline Actividad & Detalle \\
\hline $\begin{array}{l}\text { Delimitar actividad } \\
\text { proceso a ser evaluado }\end{array}$ & $\begin{array}{l}\text { Se debe centrar en una sola actividad o propósito a ser } \\
\text { evaluada, con el fin de sesgar sus hallazgos. }\end{array}$ \\
\hline $\begin{array}{l}\text { Realización de encuestas } \\
\text { multipropósito }\end{array}$ & $\begin{array}{l}\text { Las encuestas deben ser enfocadas en medir la calidad, } \\
\text { tiempos de atención, satisfacción del ciudadano, imagen } \\
\text { institucional, eficiencia de los funcionarios y dentro de lo } \\
\text { posible se pueda delimitar las tareas del proceso. }\end{array}$ \\
\hline Determinación de mudas & $\begin{array}{l}\text { Las mudas son todo aquello que consume recursos y no } \\
\text { aporta valor para el cliente y los procesos, actividad que se } \\
\text { considere inútil o innecesaria. (Ahuja Sánchez, 2016). Las } \\
\text { mudas se clasifican en: } \\
\text { M. de Defectos, M de Movimientos, M de Procesamiento, } \\
\text { M. de Espera, M. de Transporte, M. de Tiempo. }\end{array}$ \\
\hline $\begin{array}{l}\text { Levantamiento de tiempos } \\
\text { de espera y atención }\end{array}$ & $\begin{array}{l}\text { Los tiempos se toman en para cada actividad cada proceso, } \\
\text { cada paso en lo posible, con esto se puede delimitar donde } \\
\text { el funcionario invierte más tiempo o tiene mayores } \\
\text { problemas. }\end{array}$ \\
\hline $\begin{array}{l}\text { Levantamiento de causas } \\
\text { para Diagrama de Ishikawa }\end{array}$ & $\begin{array}{l}\text { En base a las tres actividades anteriores de los resultados } \\
\text { de las encuestas, detección de mudas y tiempos de espera y } \\
\text { atención se realiza el diagrama de Ishikawa o espina de } \\
\text { pescado }\end{array}$ \\
\hline Diagrama de Pareto & $\begin{array}{l}\text { Con el paso anterior se van a encontrar un sin número de } \\
\text { problemáticas, pero para saber dónde enfocarse es } \\
\text { recomendable aplicar el diagrama de Pareto que tiene como } \\
\text { premisa que resolviendo el } 20 \% \text { de los problemas se } \\
\text { solventan el } 80 \% \text { de los inconvenientes. }\end{array}$ \\
\hline $\begin{array}{l}\text { Delimitación de estrategias e } \\
\text { indicadores }\end{array}$ & $\begin{array}{l}\text { Las estrategias e indicadores ser recomienda sea realizado } \\
\text { en la planificación conjuntamente con los resultados } \\
\text { obtenidos y basados en la normativa propia de la } \\
\text { institución. }\end{array}$ \\
\hline
\end{tabular}

Fuente: Elaboración propia 
Hacer: En esta etapa o fase simplemente es realizar las actividades detalladas en la planificación.

Verificar: Mediante los indicadores y demás metodologías que se pueda utilizar según el ámbito de aplicación de la institución se analizan los resultados obtenidos.

Actuar: con los resultados se deben realizar correctivos necesarios para mejorar esos indicadores y analizar donde se está teniendo los problemas, ya sean con un funcionario, con una actividad, con un espacio físico, etc., pueden existir un sin número de escenarios que reflejen el modelo. Pero lo importante en esta etapa es identificar, corregir y volver a mejorar es decir volver al paso uno de la planificación.

\section{Metodología 5's}

En cada fase del ciclo Deming (PHVA), se debe utilizar la metodología 5's, las cuales fueron analizadas de manera individual en la tabla 3. La utilización de esta herramienta de calidad es de suma importancia ya que como se vio es un proceso de auto disciplina que busca priorizar las actividades, mantener un orden en nuestro espacio de trabajo, así como una limpieza adecuada, esto con el objetivo de ser más productivos.

\section{Estrategias $e$ indicadores}

Estos no se pueden delimitar dentro de un modelo, ya que los mismos deben ser plasmados según las necesidades y escenarios por los que atraviese la institución. Pero se recomienda que por lo menos se establezcan indicadores de:

- Satisfacción al cliente

- Productividad del personal

Adicional a esto se recomienda la herramienta de calidad como es diagramas de control para evidenciar los cambios, estos pueden ser levantados mediante la cantidad de trámites, o los tiempos de realización, tanto las estrategias como los indicadores deben ser realizados al inicio y al final de la aplicación del modelo, recordando que es un modelo cíclico y debe ser enfocado a la mejora continua.

\section{Satisfacción al cliente (Salidas)}

La aplicación del modelo debe dar como resultado una mejora que puede ser bajo diferentes enfoques todo dependerá de las estrategias e indicadores que se plantearon en la planificación. Podemos generalizar dentro del sector público en algunos resultados como son:

- Mejora de la imagen institucional.

- Control de tiempos de atención como de espera.

- Disminución de tiempo ocioso por parte de los funcionarios.

- Mejora en el lucro de la infraestructura

- Ahorro de recursos como suministros.

- Mitigación de corrupción, referente a tramitadores (dependerá de la institución). 
- Incremento en la eficiencia y eficacia de la producción, entre otros.

Como se evidencia los beneficios pueden ser varios, todo dependerá de que tan bien sea aplicado el modelo de mejora continua.

\section{Conclusiones.}

- La percepción de los servicios públicos según indicador del INEC es de 6 sobre 10, ponderación que denota una falta de calidad en los productos o servicios entregados por parte de las instituciones públicas. La calidad es esperada tanto por entidades públicas como privadas, las personas esperan recibir productos o servicios de calidad como respaldo de los impuestos pagados, caso contrario perciben un mal servicio y degrada la imanen institucional.

- Los gobiernos plantean normativas e instituciones encargadas para el control y mejora de la calidad en los servicios públicos, es por esto que en Ecuador se implementa la metodología de Gobierno por Resultados, pero este ha quedado solo como una herramienta de reporte de indicadores que no genera cambios, por lo que se es necesario impulsarlo con el modelo propuesto.

- El modelo de mejora continua busca enmarcar al GPR como estructura de varias herramientas de calidad a ser utilizadas en las instituciones, pero sin que esto afecte a la parte de presupuesto ya que como es entendible los recursos son limitados.

- Utilizando el modelo de calidad para mejorar la eficiencia se busca que los funcionarios creen una auto disciplina enfocada en la productividad, por lo cual pasan por un proceso de planificar lo que van a realizar, enfocados en un orden de prioridades, hacer lo planificado, autocriticarse mediante indicadores de producción y buscar estrategias para mejorar.

\section{Referencias bibliográficas.}

Asamblea Constituyente. (2008). Constitución del Ecuador. Asamblea Nacional Constituyente.

Becerra, J. (10 de 07 de 2013). Genesis. Obtenido de http://genesis.uag.mx/posgrado/revistaelect/calidad/cal012.pdf.

Camisón, C., Cruz, S., \& González, T. (2006). Gestión de la Calidad: conceptos, enfoques, modelos y sistemas. Madrid: Pearson Educación S.A.

Casanova Villalba, C. I. (2014). Análisis operativo y financiero al centro de atención ambulatoria y sus implicaciones en la unificación patrimonial con el hospital del seis en Santo Domingo de los Tsáchilas. (Bachelor's thesis, CIENCIAS 
ADMINISTRATIVAS FACULTAD: INGENIERÍA EN FINANZAS Y AUDITORIA CPA).

Casanova Villalba, C. I. (2018). Análisis y mejoramiento de la eficiencia del proceso de emisión de licencias de la agencia nacional de tránsito, Santo Domingo de los Tsáchilas. (Master's thesis, PUCE).

Colunga, C. (1994). Los costos de calidad. Universitaria Potosina.

Herrera Sanchez, M. J. (2014). Análisis de alternativas de inversión para las personas beneficiarias del crédito al bono de desarrollo humano asociativo en Santo Domingo. (Bachelor's thesis, CIENCIAS ADMINISTRATIVAS FACULTAD: INGENIERÍA EN FINANZAS Y AUDITORIA CPA).

Herrera Sánchez, M. J. (2018). Mejoramiento de los procesos del área técnica de la unidad administrativa provincial de la Agencia Nacional de Tránsito de Santo Domingo de los Tsáchilas y su impacto en la satisfacción del usuario. (Master's thesis, PUCE).

Imai, M. (1991). KAIZEN, la clave de la ventaja competitiva japonesa. México: Compañía Editorial Continental S.A. (CECSA).

Imai, M. (2012). Gemba Kaizen. Japon: McGraw-Hll.

Instituto Nacional de Estadística y Censo. (2020). Boletín Técnico 01-2020 Encuesta Nacional Multiproposito de Hogares. Instituto Nacional de Estadística y Censo. Obtenido de https://www.ecuadorencifras.gob.ec/documentos/webinec/Multiproposito/2019/201912_Boletin\%20Tecnico_Multiproposito.pdf

Instituto Nacional de Estadísticas y Censos. (2018). Fiche Metodológica - Encuesta multiproposito de Hogares. Instituto Nacional de Estadísticas y Censos.

Ishikawa, K. (1986). ¿Qué es el control total de calidad? La modalidad japonesa. Bogotá: Norma.

ISO 9000:2015. (2015). Sistemas de gestión de la calidad — Fundamentos y vocabulario. Ginebra: Secretaría Central de ISO.

Rueda López, N. (2011). La eficiencia y su importancia en el sector público. eXtoikos, 3847.

Secretaría Nacional de la Administración Pública. (2011). Normativa técnica de implementación y operación de la metodología y herramientas de GPR. Quito. 
Subsecretaría de Calidad en el Servicio Público. (2020). Guía Metodológica para la aplicación de la norma técnica de los mecanismos de calificación del servicio. Subsecretaría de Calidad en el Servicio Público.

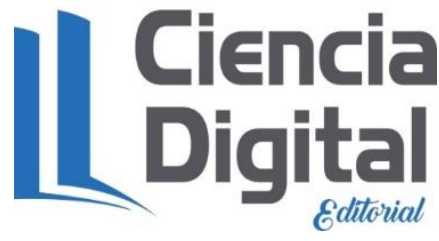




\section{PARA CITAR EL ARTÍCULO INDEXADO.}

Casanova Villalba, C. I., Herrera Sánchez, M. J., Navarrete Zambrano, C. M., \& Ruiz López, S. E. (2021). Modelo de calidad para el mejoramiento de la eficiencia en las instituciones públicas del Ecuador. Ciencia $\quad$ Digital, $\quad 5(1), \quad$ 15-29. https://doi.org/10.33262/cienciadigital.v5i1.1516

\section{LCiencia}

El artículo que se publica es de exclusiva responsabilidad de los autores y no necesariamente reflejan el pensamiento de la Revista Ciencia Digital.

El artículo queda en propiedad de la revista y, por tanto, su publicación parcial y/o total en otro medio tiene que ser autorizado por el director de la Revista Ciencia Digital.
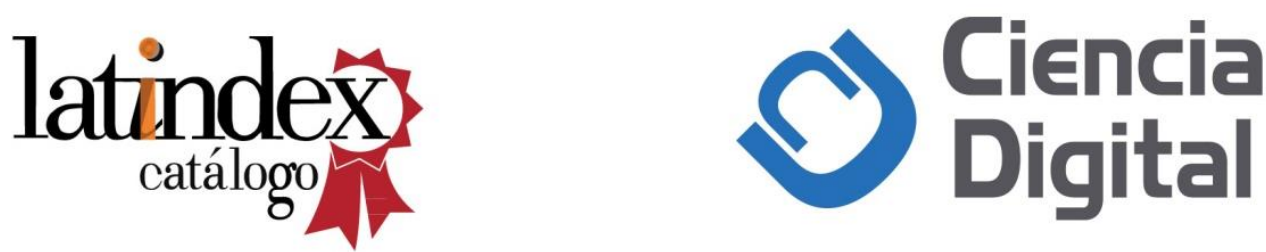